\title{
Segmental colitis complicating diverticular disease
}

\author{
GUIDO MA VAN ROSENDAAL MD FRCPC, MELVIN A ANDERSEN MD FRCPC
}

GMA VAN RosendaAl, MA ANDERSEN. Segmental colitis complicating diverticular disease. Can J Gastroenterol 1996; 10(6):361-364. Two cases of idiopathic colitis affecting the sigmoid colon in elderly patients with underlying diverticulosis are presented. Segmental resection has permitted close review of the histopathology in this syndrome which demonstrates considerable similarity to changes seen in idiopathic ulcerative colitis. The reported experience with this syndrome and its clinical features are reviewed.

Key Words: Diverticulosis, Idiopathic colitis, Inflammatory bowel disease

\section{Diverticulite compliquée de colite segmentaire}

RÉSUMÉ : Deux cas de colite idiopathique affectant le sigmoïde chez des sujets âgés souffrant de diverticulose sous-jacente sont présentés ici. La résection segmentaire a permis un examen attentif de l'histopathologie de ces syndromes qui démontrent une similarité considérable avec les anomalies observées dans la colite ulcéreuse idiopathique. L'expérience signalée avec ce syndrome et ses caractéristiques cliniques sont présentées ici.
A few reports have identified a distinctive idiopathic colitis that occurs segmentally in areas of sigmoid colon involved with diverticular disease (1-5). Documentation of the pathological changes in this condition has been limited because tissue obtained from reported patients has been restricted to biopsies obtained endoscopically. We describe two patients with this condition, one of whom underwent segmental resection, allowing more extensive examination of the underlying histopathology.

\section{CASE 1 PRESENTATION}

In 1987 a 67-year-old man reported that his stool had become softer, more frequent and intermixed with dark blood or covered with bright red blood. His weight had fallen about $2.5 \mathrm{~kg}$. He had 'parapsoriasis' in the early 1970s. Fibreoptic sigmoidoscopy and barium enema revealed moderately severe diverticular disease with patchy erythema and edema limited to the area of diverticular disease (Figure 1). The dis- tal colon appeared normal. Stool microscopy showed many Blastocystis hominis, Entamoeba hartmanni, Endolimax nana and Dientamoeba fragiles trophozoites. He was treated initially with 5-aminosalicylic acid (5-ASA) and metronidazole, with resolution of the symptoms and endoscopic findings.

From 1988 to 1992, recurrent episodes of diarrhea and hematochezia were treated successfully with 5-ASA and corticosteroid enemas. Sigmoidoscopic appearance remained similar to that at presentation. Repeated stool microscopy was negative for ova and parasites. He continued on 5ASA and did not seek further gastroenterological care until April 1994.

In mid-1991 the patient developed a severely pruritic rash; eczema was diagnosed and treated with topical corticosteroids during 1992. Methotrexate was transiently used in early 1993. Azathioprine was prescribed without improvement, and he was evaluated in another centre where treat-

Department of Medicine, Health Sciences Centre, Calgary, Alberta

Correspondence: Dr GMA Van Rosendaal, Department of Medicine, Room 1410, Health Sciences Centre, 3330 Hospital Drive NW, Calgary, Alberta T2N 0T5. Telephone 403-220-4500, fax 403-283-3028, e-mail gurosend@acs.ucalgary.ca

Received for publication July 28, 1995. Accepted November 21, 1995 


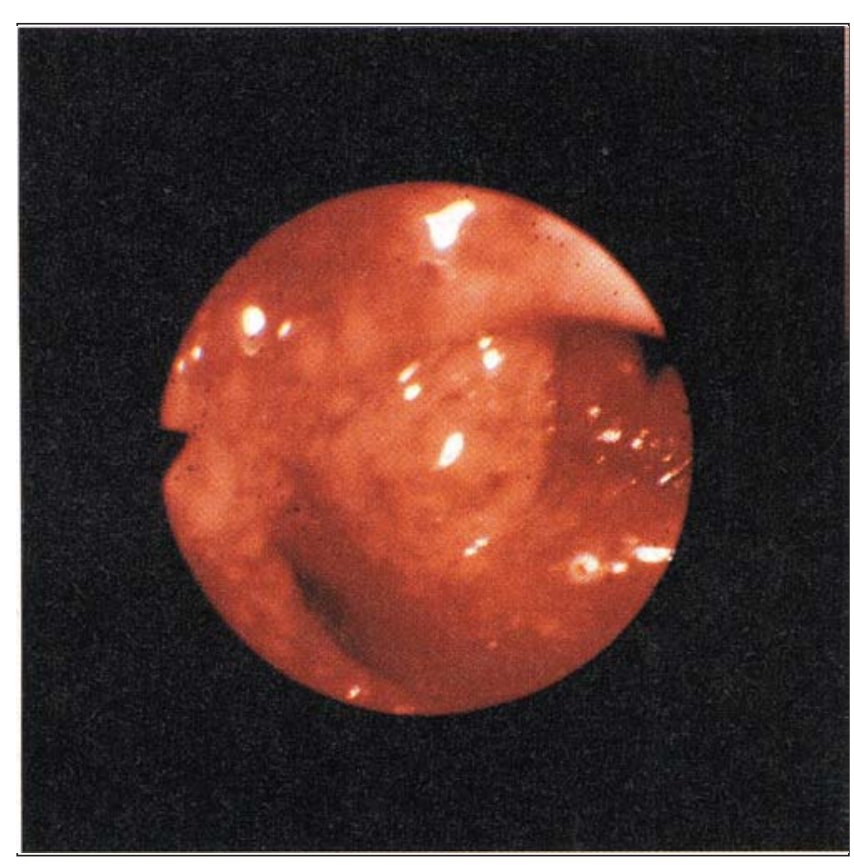

Figure 1) Sigmoidoscopic appearance of widespread erythema in the sigmoid colon

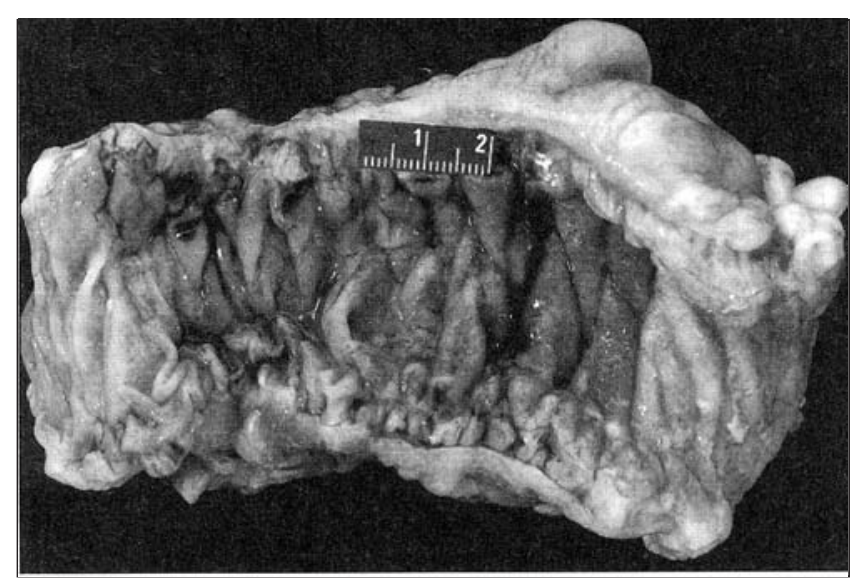

Figure 2) Resected specimen showing central congestion, patchy ulceration and exudate over the mucosal surface. Diverticular openings are obscured by mucosal folds

ment with cyclosporine and prednisone for 'chronic eczematous dermatitis' was begun. In April 1994 he was floridly cushingoid and taking olsalazine sodium. Previous withdrawal of 5-ASA had been associated with worsening of the dermatitis but because his skin condition could have been related to 5-ASA sensitivity, olsalazine sodium was stopped and he was tapered off corticosteroids. The dermatitis gradually improved but rectal bleeding and diarrhea worsened, with a fall in hemoglobin. Sigmoidoscopy showed a normal distal colon. At approximately $20 \mathrm{~cm}$, edema, erythema and ulceration were evident in the narrowed diverticular segment which would not allow passage of a gastroscope. Stool microscopy was negative for ova and parasites. Segmental resection of the sigmoid colon was undertaken in September 1994, with resolution of the diarrhea and hematochezia.

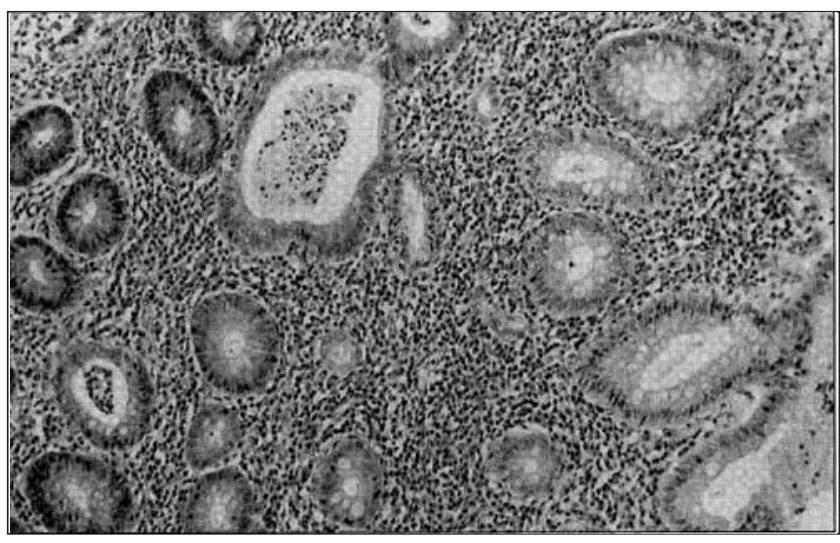

Figure 3) Moderately severe chronic inflammatory infiltrate in the lamina propria and several crypt abscesses (hematoxylin and eosin $\mathrm{x102}$ )

Postoperatively he experienced transient lower abdominal discomfort, lassitude and weight loss, but no fevers, sweats or leukocytosis. He ceased using cyclosporine on his own initiative and experienced a diathesis that included malaise, weakness, lymphadenopathy, severe depression, thrombocytosis, anemia, eosinophilia, and elevation of alkaline phosphatase and erythrocyte sedimentation rate (ESR). Abdominal ultrasound and computed tomographic scans were negative. Over approximately three months these symptoms and laboratory abnormalities gradually normalized without specific therapy. His skin condition gradually improved. Fibreoptic sigmoidoscopy 11 weeks postoperatively revealed minor erythema around the surgical anastomosis with normal mucosa above and below. One year later he is well, without gastrointestinal symptoms and with a mild, ongoing dermatitis.

\section{CASE 2 PRESENTATION}

A 78-year-old man was evaluated in November 1994 for hematochezia - red blood was intermixed with his stool, which was otherwise normal. He complained of fatigue and a sense of rectal pressure. He had undergone sigmoidoscopy in 1987 because of constipation with thin calibre stools and weight loss. At that time diverticular disease with marked patchy erythema involving the sigmoid colon and sparing of the rectum was identified. Histopathology revealed 'nonspecific chronic inflammation' and a course of 5-ASA was given. He remained free from intestinal symptoms aside from chronic constipation and an intermittent foreign body or rectal pressure sensation. Fibreoptic sigmoidoscopy in 1982 revealed diverticular disease without any mucosal change.

He received warfarin sodium for chronic atrial fibrillation and was on long term treatment for chronic congestive heart failure, asymptomatic gallstones and osteoarthritis. Complete blood cell count, electrolytes, blood urea nitrogen, blood sugar, antinuclear antibody, thyroid-stimulating hormone and ESR were normal.

Changes identified at colonoscopy to the cecum were limited to the sigmoid colon where severe diverticular disease was identified. Within the involved area, between 20 and 


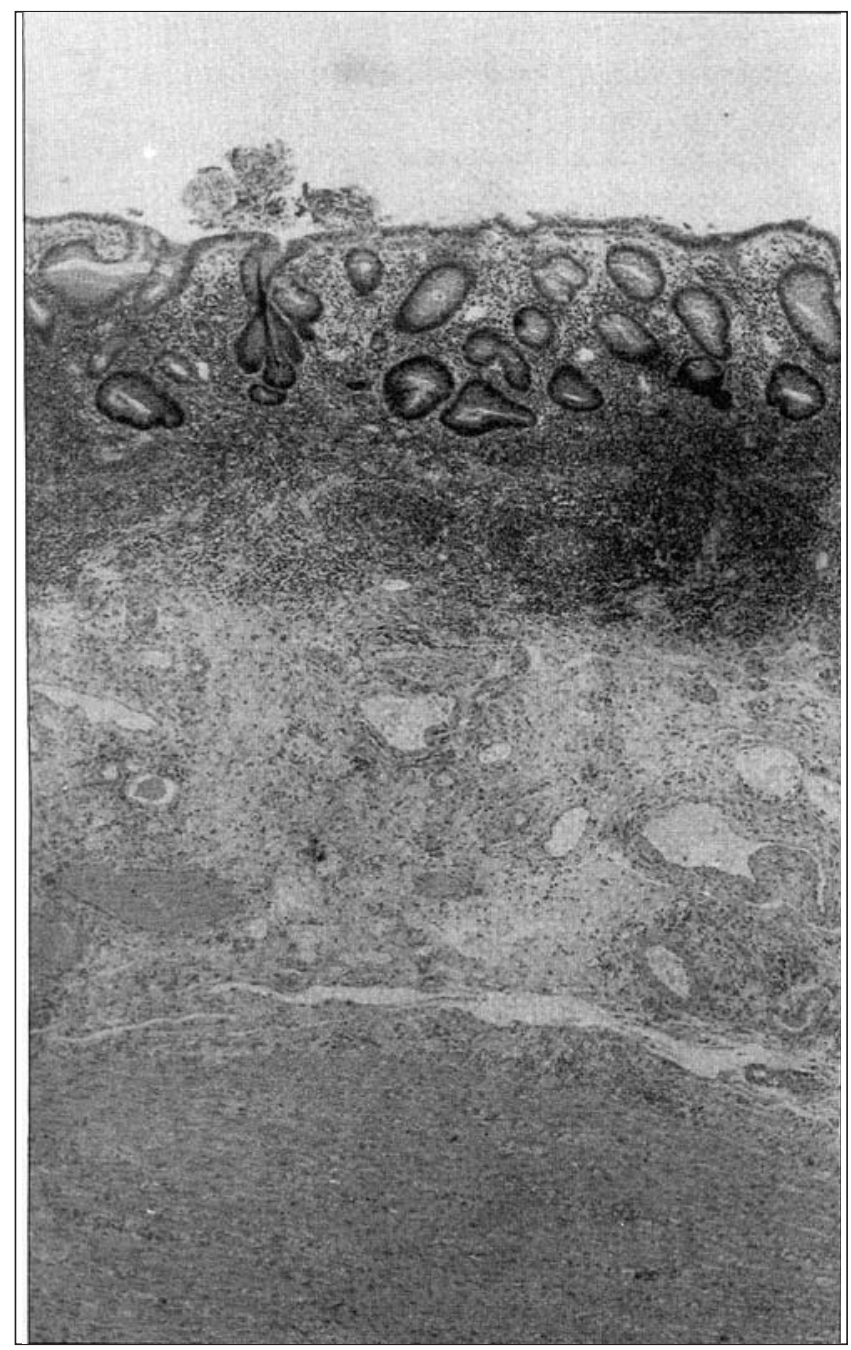

Figure 4) Changes similar to those in Figure 3 are seen except that there is a chronic inflammatory infiltrate beneath the muscularis mucosa located in the superficial portion of the widened submucosa (hematoxylin and eo$\sin \times 24$ )

$40 \mathrm{~cm}$ from the anus, there was patchy erythema not contiguous with the diverticulae.

5-ASA was given, with initial resolution of bleeding and marked worsening of constipation. A few weeks later hematochezia recurred despite ongoing therapy with 800 mg 5-ASA qid. Sigmoidoscopy revealed colitis limited to a segment of less than $10 \mathrm{~cm}$ in the sigmoid colon.

\section{PATHOLOGY OF CASE 2}

Gross description: The external aspect of the resected colon was unremarkable. From the mucosal aspect an 11.0 to $12.0 \mathrm{~cm}$ segment revealed patches of loosely adherent yellowish exudate 0.1 to $0.3 \mathrm{~cm}$ in diameter. In addition, the middle segment $(9.0 \mathrm{~cm})$ showed mucosal congestion. The remaining mucosa appeared normal. Diverticular openings scattered throughout, including in the noninflamed mucosa, were noted (Figure 2).

Microscopic description: Sections from the grossly abnormal areas showed a predominantly mucosal inflammatory process consisting of a moderate number of lymphocytes and

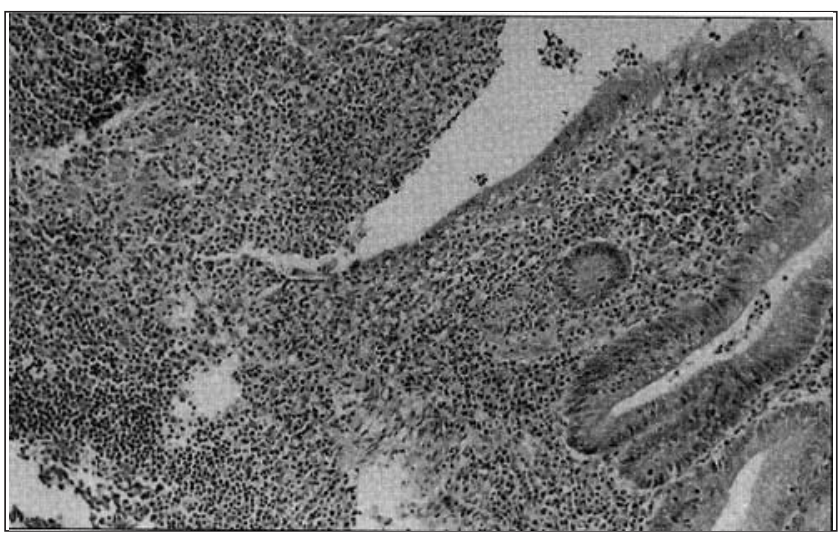

Figure 5) Section from ulcer edge showing an undermining ulceration, re-epithelialization and inflammatory infiltrate (hematoxylin and eosin $x 102)$

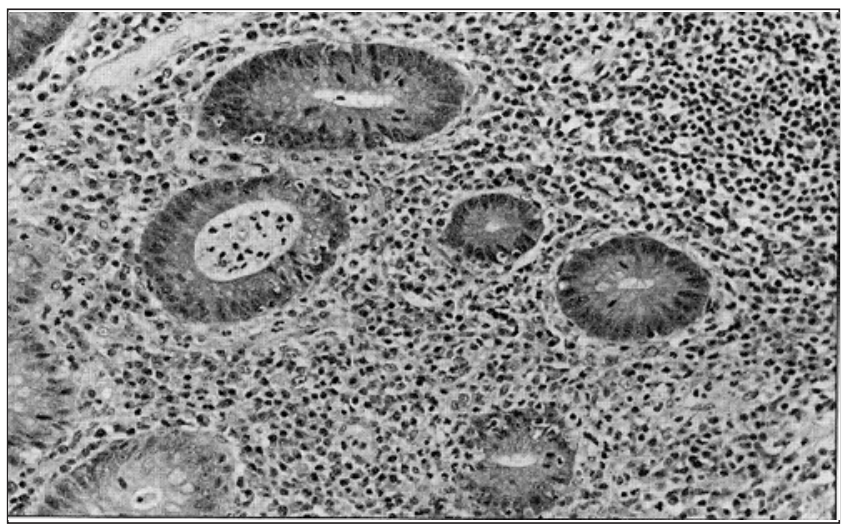

Figure 6) Photomicrograph showing chronic lamina propria inflammation - a small crypt abscess on the left and acute cryptitis at the bottom (hematoxylin and eosin $x 102$ )

plasma cells within the lamina propria with occasional eosinophils and polymorphs, and scattered cryptitis and crypt abscess formation (Figure 3). The submucosa was widened with edema, telangiectasia and infrequent focal lymphocytic collections (Figure 4). In occasional areas there was somewhat more diffuse band-like chronic inflammatory reaction along the muscularis mucosa. The muscularis propria revealed only occasional inflammatory cells, and in the subserosal fat there were some areas of fibrosis and nodular lymphocytic inflammation. Ulcerations were mainly nondescript with acute inflammatory to granulation tissue bases, frequently with re-epithelialization and occasionally showing an 'undermining' architecture (Figure 5). No section revealed evidence of 'fissuring' ulceration or granulomata. Sections from the grossly normal area demonstrated normal histology without evidence of inflammation or crypt architectural distortion. The diverticulae were all associated with chronic inflammation, focal cryptitis and crypt abscess formation (Figure 6). Ulceration was present in one section. A diverticulum from the grossly normal segment also showed inflammatory change and one crypt abscess was present. There was no evidence of vasculitis. The vessels demon- 
strated only focal subintimal fibrosis and no significant narrowing.

The patient's previous sigmoid biopsies were reviewed. All showed a relatively uniform histology consisting of a mild to moderate chronic inflammatory reaction consisting of lymphocytes and plasma cells with occasional eosinophils and focal acute cryptitis. No crypt abscesses or ulcerations were identified. Histopathology in the second patient was similar to that seen in the resected segment and superficial biopsies of the first patient.

\section{DISCUSSION}

We believe that these cases are representative of an idiopathic colitis syndrome associated with diverticular disease that has been identified in a few published case reports with limited pathological descriptions. Our first case had a particularly severe form of this syndrome that was responsive to corticosteroid and cyclosporine given for a progressively worsening skin condition (we believe 5-ASA sensitivity was a major contributing factor). Because of major side effects from all effective medicines, surgical resection was undertaken, with apparent cure of the colitis. The parasites transiently identified early in his course are generally considered to be nonpathogenic, aside from $B$ hominis, which appears to be of low pathogenicity, causing a mild diarrheal illness in some individuals (6-9). Although one report suggests that it was responsible for a case of colitis (10), another study failed to identify any pathogenicity in patients with inflammatory bowel disease (11). Because the colitis persisted in our pa-

\section{REFERENCES}

1. Peppercorn MA. Drug-responsive chronic segmental colitis associated with diverticula: a clinical syndrome in the elderly. Am J Gastroenterol 1992;87:609-12.

2. Sladen GE, Filipe MI. Is segmental colitis a complication of diverticular disease? Dis Colon Rectum 1984;27:513-4.

3. Cawthorn SJ, Gibbs NM, Marks CG. Segmental colitis: A new complication of diverticular disease. Gut 1983;24:A500.

4. Friedman LS. The special problem of colitis in the elderly: expanding the spectrum. Gastroenterology 1992;103:1984-5.

5. Polit SA. Chronic segmental colitis in association with diverticulosis: A clinical syndrome in the elderly? J Am Geriatr Soc 1993;41:1155-6. (Lett) tient following eradication of B hominis, it appears that its presence was coincidental, although some form of triggering may have occurred.

The absence of granulomas and the superficial character of the inflammation weigh against the diagnosis of Crohn's disease, although they do not exclude it. The absence of changes in the vasculature argue against a vascular basis. An intermittent low-flow state may be considered as a cause although the dramatic symptomatic and sigmoidoscopic responses to 5-ASA treatment would be surprising if such a process were responsible.

Individuals with this idiopathic colitis syndrome present with a combination of symptoms, including diarrhea, hematochezia and the passage of mucous. They are of advanced age; the youngest reported patient was 60 years old. The disease occurs in segments of sigmoid colon involved with diverticulosis, is patchy (early on, at least) and does not appear to originate within the diverticulae. The course varies in severity, may be chronically recurrent and appears to be responsive to medications used to treat idiopathic inflammatory bowel disease. The distal colon is spared. Histopathologically, the appearance is more similar to idiopathic ulcerative colitis than to granulomatous colitis.

It is unclear whether this syndrome is distinct from 'traditional' idiopathic inflammatory bowel disease or whether the presence of diverticulae in an older population somehow modifies the pathophysiology seen in younger patients with idiopathic colitis. Further study of this syndrome may shed light on the pathogenesis of inflammatory bowel disease.

6. Senay H, MacPherson D. Blastocystis hominis: Epidemiology and natural history. J Infect Dis 1990;162:987-90.

7. Udkow MP, Markell EK. Blastocystis hominis: prevalence in asymptomatic versus symptomatic hosts. J Infect Dis 1993;168:242-4.

8. Zierdt CH. Blastocystis hominis - past and future. Clin Microbiol Rev 1991;4:61-79.

9. Zuckerman MJ, Watts MT, Ho H, et al. Blastocystis hominis infection and intestinal injury. Am J Med Sci 1994;308:96-101.

10. Russo AR, Stone SL, Taplin ME, et al. Presumptive evidence for Blastocystis hominis as a cause of colitis. Arch Intern Med 1988;148:1064.

11. Nagler J, Brown M, Soave R. Blastocystis hominis in inflammatory bowel disease. J Clin Gastroenterol 1993;16:109-12. 


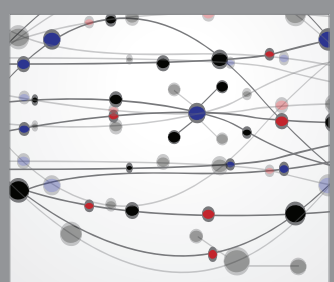

The Scientific World Journal
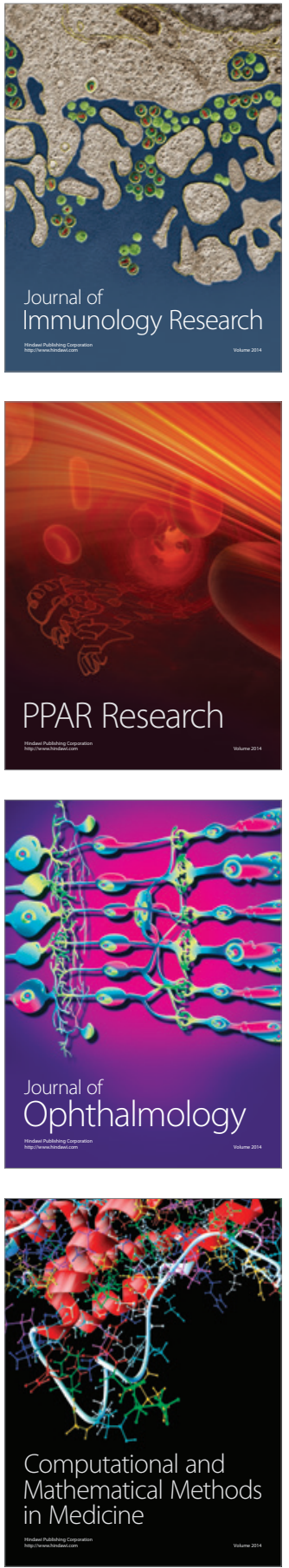

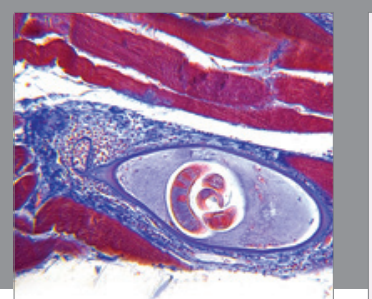

Gastroenterology Research and Practice

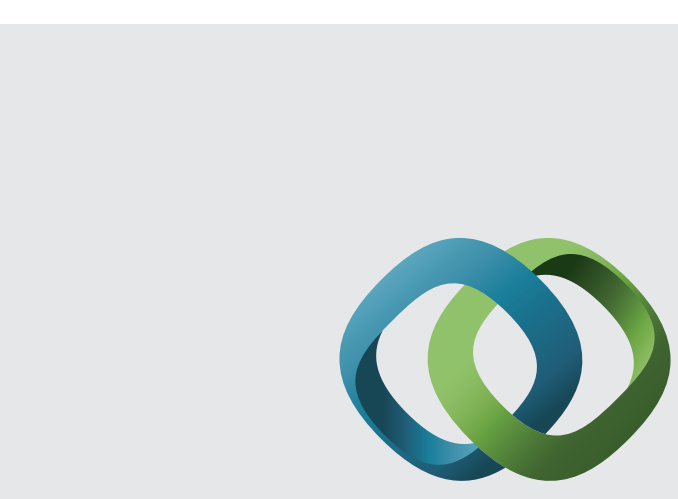

\section{Hindawi}

Submit your manuscripts at

http://www.hindawi.com
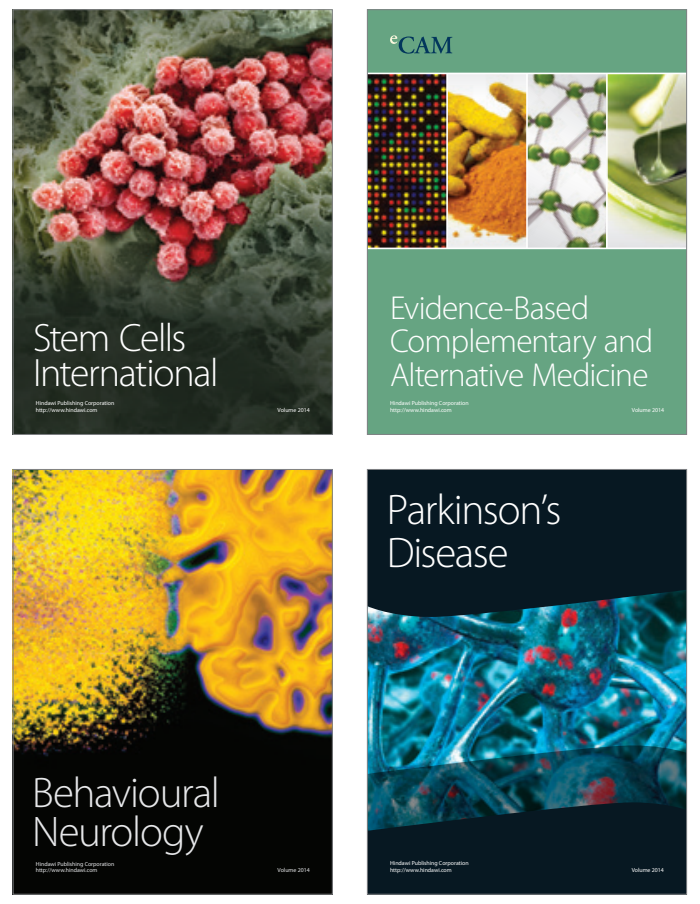
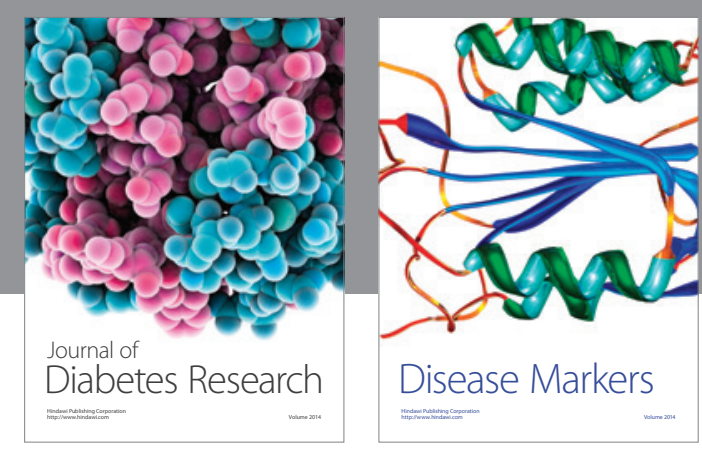

Disease Markers
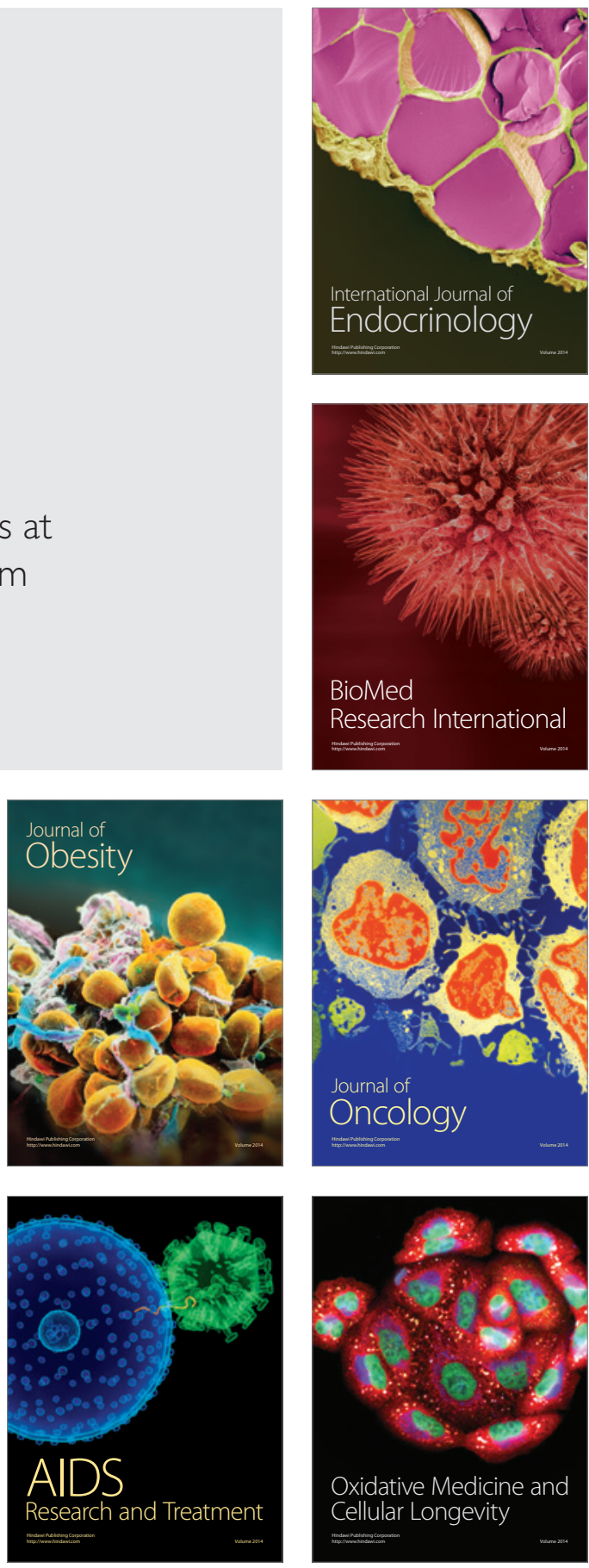